\title{
Optimisation de la couverture du risque de change par l'algorithme génétique
}

\section{Optimization of foreign exchange risk hedging by the genetic algorithm}

\author{
Mustapha Bouchekourte ${ }^{1}$, Sara Rhouas ${ }^{2}$, Norelislam El Hami \\ ${ }^{1}$ Laboratoire de Recherche en Sciences de Gestion des Organisations, Ecole Nationale de Commerce et de Gestion, \\ Université Ibn Tofail, Kénitra, Maroc \\ ${ }^{2}$ Laboratoire Sciences et Ingénierie, Ecole Nationale des Sciences Appliquées, Université Ibn Tofail, Kénitra, Maroc
}

\begin{abstract}
RÉSUMÉ. Les chercheurs et développeurs scientifiques ont aujourd'hui une énorme quantité de données à traiter. lls ont besoin des solutions efficaces et rapides pour traiter et modéliser ces données. C'est pour cela qu'ils ont développé une métaheuristique basée sur l'évolution génétique naturelle. L'algorithme génétique ne prend pas en compte toutes les alternatives, mais c'est une technique rapide pour trouver une solution décente aux problèmes caractérisés par un flux importants de données. Dans de nombreux domaines, les données doivent être traitées dans le plus bref délai et dans cet article nous avons traité une nouvelle façon de trouver l'optimum du ratio de couverture par les contrats à terme, pour objectif de diminuer le risque présent sur le marché des produits dérivés, relatif aux fluctuations des prix de n'importe quel actif sous-jacent des contrats à terme soit des matières premières, de taux de change ou bien d'indices boursiers... Dans notre cas nous avons choisi le pétrole comme exemple d'application sur la fluctuation des prix des matières premières sur un horizon de 10 ans en appliquant le modèle de couverture de minimisation de la variance d'Ederington comme fonction objectif de notre algorithme d'optimisation génétique sur le logiciel MATLAB.
\end{abstract}

ABSTRACT. Researchers and scientific developers today have a huge amount of data to process, they need a solution as quickly as possible, which is why they have developed this metaheuristic based on natural genetic evolution. The genetic algorithm does not take into account all the alternatives, but it is a quick technique to find a decent solution to problems with a lot of data. In many areas, data must be processed as quickly as possible and in this article we have discussed a new way to find the optimum coverage ratio for futures contracts, with the objective of decreasing the risk that one must face in the derivatives market, against fluctuations in the prices of any underlying asset of the futures contracts either commodities, exchange rates or stock market indices ...., in our case we have chosen oil as an example of an application on the fluctuation of commodity prices of a 10-year data margin, by applying the Ederington variance minimization hedge model as an objective function of our genetic optimization algorithm on MATLAB software.

MOTS-CLÉS. Métaheuristiques, Algorithme génétique, risque de change.

KEYWORDS. Metaheuristic, Genetic algorithm, foreign Exchange risk.

\section{Introduction}

Le commerce international est le cœur de l'économie mondiale et représente $60 \%$ du produit intérieur brut dans le monde entier. Les flux transactionnels à l'international ont permis à plusieurs entreprises d'établir de nombreuses relations d'affaires. Bien que le commerce international ait permis de développer l'économie de plusieurs pays, celui-ci a multiplié les canaux de transmission de risque de change que plusieurs entreprises et multinationales doivent gérer attentivement pour minimiser les moins-values que pourraient résulter d'une mauvaise quantification et gestion de cette catégorie de risque.

Les entreprises évoluent dans un environnement financier qui leur fournit des outils spécialisés et des plans d'action, mais les place également sous de forts risques financiers. C'est ce qui permet de définir la finalité de la gestion financière ou de la finance d'entreprise en fonction des opportunités et des limites imposées par l'environnement financier. Chaque partie prenante doit non seulement être consciente des risques et menaces qui peuvent nuire sur ses activités, mais également être en mesure d'évaluer la probabilité que ces risques se produisent ainsi que leur gravité potentielle s'ils le font. 
De ce fait, chaque entreprise doit analyser et évaluer son risque financier dans le but de pouvoir prendre des mesures correctives en fonction de la gravité de ces risques.

Ce risque financier peut prendre plusieurs formes. On trouve par exemple le risque de contrepartie lorsque le contrat conclu entre les parties concernées ne respectent pas leurs engagements comme par exemple les banques qui sont aussi concernées par ce risque que leurs clients ne remboursent pas leurs dettes ou crédits, nous avons aussi le risque du marché qui se résume sur le risque de perte adossée aux placements financiers induits par des variations de prix défavorables et qui contient le risque de taux qui concerne les produits de taux, et aussi le risque le plus fréquent dans la gestion financière à savoir le risque de change qui fait l'objet de notre recherche et qui fait référence à la possibilité de variations des taux de change entre le moment de la passation du contrat et la période du paiement effectif. Le montant reçu par l'exportateur peut être inférieur ou supérieur à celui convenu au départ, en fonction des fluctuations du marché des devises, ce qui pourrait diminuer considérablement sa marge sur les produits exportés. C'est un risque de fluctuation de change ou aussi bien de cours de la matière première quelle qu'elle soit le bien approvisionné [LIBO 21- AND 21].

La gestion du risque de change se fait de plusieurs manières, parmi ces méthodes, on trouve l'utilisation du contrat à terme qui est un engagement dans lequel deux parties se mettent d'accord d'acheter ou de vendre une certaine quantité d'un bien à un prix et une date d'échéance prédéterminés, et qui permet de diminuer l'inquiétude auprès des commerçants concernant l'évolution de la volatilité des prix de ces biens. Ce contrat à terme est utilisé pour couvrir un portefeuille contre les fluctuations futures du marché en prédisant les changements futurs d'un actif sous-jacent et permet à plusieurs commerçants et entrepreneurs de profiter de la hausse ou de la baisse des prix, pour reduire le risque survenant des biens physiques, et permet également de contribue à l'amélioration de la performance du portefeuille [DON 05- BEA 15].

L'objectif principal de cet article est de minimiser le risque de change en utilisant l'algorithme génétique d'optimisation pour résoudre ce prolème. Dans la première partie de cet article nous allons définir la métaheuristique évolutionnaire génétique. La deuxième partie sera consacrée à étudier et mieux définir la fonction objectif de notre problèmatique. Et la dernière partie sera réservée à étudier la performance de l'algorithme génétique de notre problème de minimisation du risque dans le marché financier.

\section{Algorithme d'optimisation}

\subsection{Algorithme génétique}

L'algorithme génétique est une métaheuristique évolutionnaire utilisée pour trouver l'optimum global des problèmes compliqués dont la solution est inconnue durant un laps de temps court, et qui a été développée par le chercheur John Holland depuis 1960.

L'algorithme génétique commence par générer aléatoirement un nombre précis d'individus dans notre programme appelé chromosome ou plus précisément $\mathrm{n}$ chaînes de caractères ou de bits et que chaque chaîne correspond à un chromosome qui sera notre population de base, et bien sûr appartenant à notre espace de recherche (figure 1) [SAI 16-MAA 16]. Chaque chromosome est généré sous forme de chaine de bits selon le nombre de variables recherchées sur notre problème à résoudre appelés gènes sur notre algorithme d'optimisation. Ensuite on évolue notre population initiale en utilisant en majorité de temps notre modélisation du problème à résoudre qui est la fonction objective ou bien la fonction fitness [LILI 21- IVAN 21]. Après on passe à l'étape de sélection qui consiste à choisir les chromosomes les plus performants de notre population et cela en utilisant l'un des deux critères ci-dessous : 
- L'ordonnancement en anglais «Ranking»; est le critère le plus simple dans la phase sélection et qui se résume en classifiant notre population par ordre de performance, le meilleur chromosome qui va réaliser notre optimisation soit de minimisation ou de maximisation avec vérification des conditions sera en premier dans notre vecteur et ainsi de suite selon notre problématique étudié.

- La roue de la fortune en anglais «Wheel Selection»; consiste à attribuer pour chaque chromosome dans notre population $\mathrm{N}$, une probabilité $P_{i}$ proportionnelle à sa fonction objective $F_{i}$ selon l'équation 1 suivante :

$$
P_{i}=\frac{F_{i}}{\sum_{j=1}^{N} F_{j}}
$$

Après nous passons à la deuxième phase de l'algorithme génétique qui est le croissement ou aussi connu en anglais «Crossover», consiste à combiner les caractéristiques ou les gènes des meilleurs chromosomes parents de notre population pour but de générer des nouveaux chromosomes en se basant sur une probabilité de croissement qui varie en majorité des problèmes à résoudre entre $70 \%$ et 95\% [XUN 11- MAR 16]. Il existe trois types de croissement dans la littérature :

- Croissement à un point consiste à choisir un point au hasard des deux parents chromosomes qui sera notre point de croissement. Les bits à droite de ce point seront échangés pour générer deux nouveaux descendants.

- Croissement à deux points consiste à choisir deux points de croissement au hasard depuis les deux parents et les échangeais entre eux.

- Croissement uniforme consiste à choisir un bit parmi l'un des parents avec une probabilité égale. Et parfois des rapports de mélange sont utilisés pour qu'un parent hérite plus de gènes que de l'autre.

Enfin nous passons à la dernière phase principale de l'algorithme qui est la mutation qui consiste de faire changer aléatoirement certaine information génétique du nouveau chromosome en se basant sur la probabilité de mutation qui varie en général entre 0,5 et 1 afin de créer la nouvelle génération, ce phénomène se répète jusqu'à atteindre le nombre de génération maximal fixé sur notre algorithme ou atteindre la performance souhaitée.

\subsection{Fonction Objectif}

Le modèle de minimisation de la variance a été le premier modèle de couverture sélective à arriver sur le marché. Jusqu'à récemment, la seule théorie de gestion du risque de change était la couverture naïve. C'est-à-dire que la position au comptant est complètement couverte. Johnson a été le premier à remarquer le désir des agents de réduire le risque par des activités de couverture, mais parce qu'il n'a pas effectué de test empirique de son modèle, il est resté dans l'ombre jusqu'aux contributions d'Ederington en 1979. Johnson a été le premier à remarquer le désir des agents de réduire le risque grâce à des activités de couverture, mais parce qu'il n'a pas effectué de test empirique de son modèle, il est resté dans l'ombre jusqu'aux contributions d'Ederington en 1979 [MIG 99-SUS 16].

Le modèle d'Ederington s'est basé sur la théorie du portefeuille d'où ce dernier contient une position fixe d'avoir au comptant et d'une position quelconque à terme, et a découvert les équations de variance de rendement $\operatorname{Var}(\mathrm{P})$ et la contrainte d'espérance de rendement $\mathrm{E}(\mathrm{P})$ formées par les positions au comptant et à terme comme indiqué dans la formule 2 suivante : 
[2] $\left\{\begin{array}{l}\operatorname{Min} \operatorname{Var}(P)=\sigma_{S}^{2}+X_{f}^{2} \cdot \sigma_{f}^{2}+2 \cdot X_{f} \cdot \sigma_{S, f} \\ \text { Contrainte : } \\ E(P)=E\left(\Delta P_{S}\right)+X_{f} \cdot E\left(\Delta P_{f}\right)\end{array}\right.$

$X_{S}$ : au comptant proportion d'actifs (avoirs) dans le portefeuille

$X_{f}$ : Proportion à terme d'actifs (avoirs) en portefeuille

$\sigma_{s}$ : variance de la variation du prix au comptant sur une période donnée

$\sigma_{f}$ : variance de la variation du prix à terme sur une période donnée

$\sigma_{\mathrm{s}, \mathrm{f}}$ : covariance de la variation des deux prix sur une période donnée

$\Delta \mathrm{P}_{\mathrm{S}}$ : variation du prix au comptant sur une période donnée

$\Delta \mathrm{P}_{\mathrm{f}}$ : variation des prix à terme sur une période donnée

Ils ont obtenu la formule 3 ci-dessous du taux de couverture optimal après avoir réécrit l'équation selon le taux de couverture en voulant trouver le ratio optimal :

$$
R=-\frac{\sigma_{\mathrm{s}, \mathrm{f}}}{\sigma_{s}^{2}}
$$

Dans cet article nous avons choisi comme fonction fitness à résoudre qui a pour minimiser le risque de change est le modèle d'Ederington et qu'en utilisant son modèle de minimisation de la variance va nous permettre de couvrir notre portefeuille du risque de change. Notre objectif principal est de chercher le taux de couverture ou bien la proportion à terme d'actifs $X_{f}$ obtenu par le programme GA qui minimise la variance de l'erreur de couverture résiduelle et la comparer avec le taux de couverture optimal de la littérature, la figure 1 résume le fonctionnement de notre algorithme génétique pour trouver notre taux de couverture optimal.

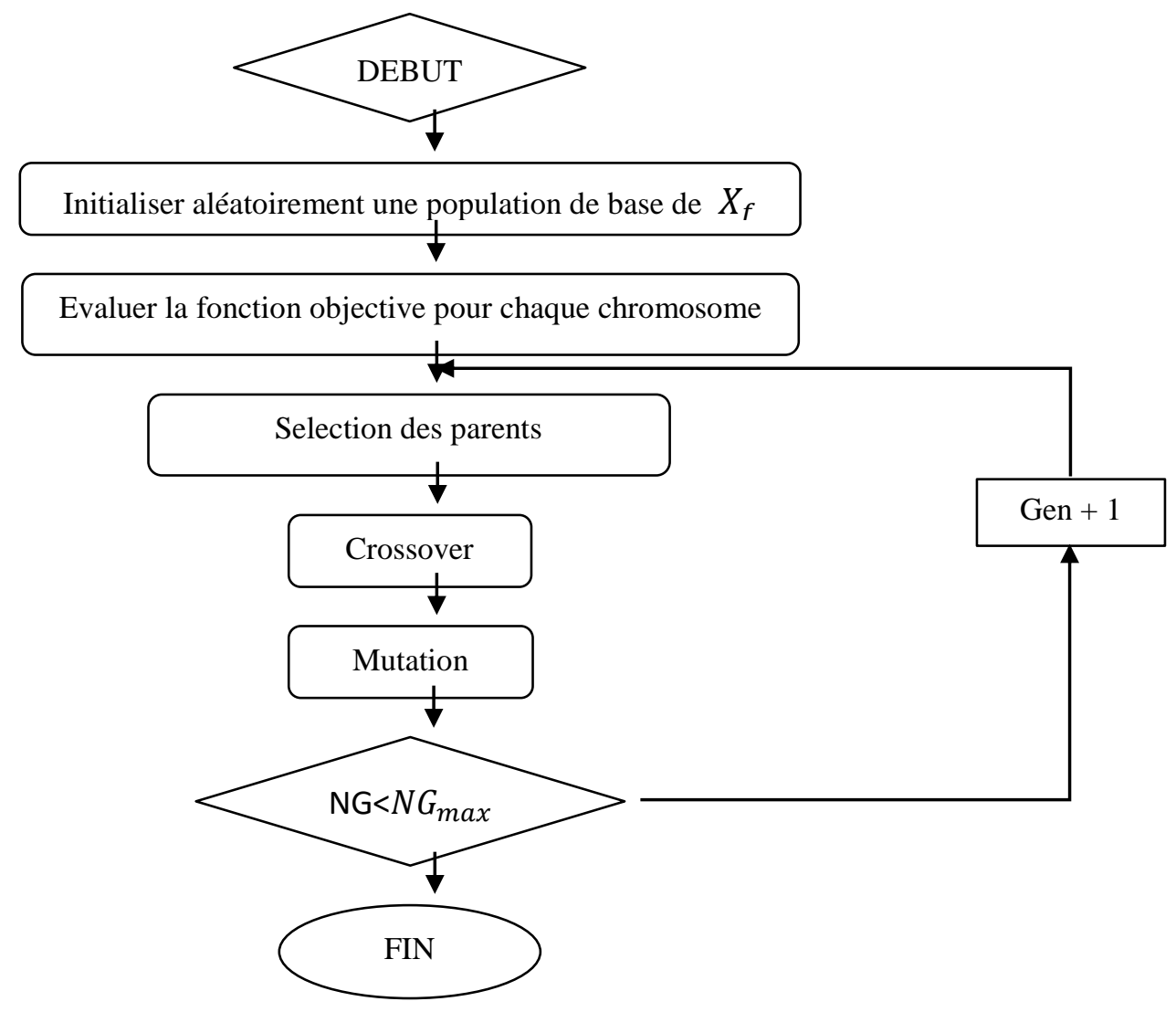

Figure 1. Organigramme du fonctionnement de l'algorithme génétique 


\section{Résultats}

\subsection{Données d'application}

Les devises ou les actifs financiers ne sont pas les seuls éléments qui caractérisent le marché financier international. Les matières premières extraites des ressources naturelles comme par exemple le blé et le soja pour les produits agricoles, ou bien l'or et l'argent dans le cas des métaux, ou aussi le pétrole dans le secteur énergétique, et qui font tous partie des éléments bruts. Ces matières premières sont produites et utiliser sous influences de divers facteurs comme le climat, la demande et aussi plusieurs autres facteurs économiques. Leurs prix sont négociés et soumis à une grande volatilité sur le marché au comptant et aussi celui à terme.

Le pétrole est l'une des matières premières que depuis les années 50 est devenu la première source d'énergie commercialisée à l'international et dont le prix sur le "marché au comptant »se base sur l'offre et la demande. De plus, les marchés à terme permettent aux producteurs de transporter des volumes importants de pétrole à un prix fixé à l'avance, se protégeant ainsi des fluctuations défavorables de prix. La volatilité des prix s'est accrue en raison de l'expansion rapide des transactions pétrolières. En temps de crise, le prix du baril a ainsi pu s'écarter considérablement des fondamentaux du marché pétrolier, à la hausse comme à la baisse.

Les données utilisées pour résoudre notre problématique sont les prix du pétrole brut sont des prix spot d'une période de 10 ans depuis l'année 2010 issu de la plateforme Bloomberg (quantités achetées pour livraison immédiate, ce qui diffère de la cotation des contrats à terme). Ce sont des prix de référence pour le marché. Cependant, certaines matières premières sont négociées sur la base de contrats à terme.

\subsection{Interprétation des résultats}

Le but de cet article est de trouver le meilleur ratio de couverture, ou la proportion de contrats à terme qui va nous permettre de réduire la volatilité de l'erreur de couverture résiduelle et donc réduire le risque. Les figures ci-dessous représentent la description des résultats du taux de couverture obtenu par l'algorithme d'optimisation génétique de couleur orange (RatioGA) comparé à celle du modèle d'Ederington de couleur bleue (Ratio) selon les équations définies dans la deuxième partie, en utilisant une fréquence de 2 semaines et d'une fréquence d'un mois (4 semaines).

L'évolution de la valeur du taux de couverture dans une fréquence de 2 semaines et d'un mois qui définit le taux de réduction du risque, et celle obtenue en utilisant l'algorithme génétique - en la comparant à celle de la littérature - sont illustrés sur les figures 2 et 3 ci-dessous. On peut clairement constater que les deux taux de couverture sont variants et que les valeurs des différents taux s'éloignent lorsque la période de la fréquence augmente. 


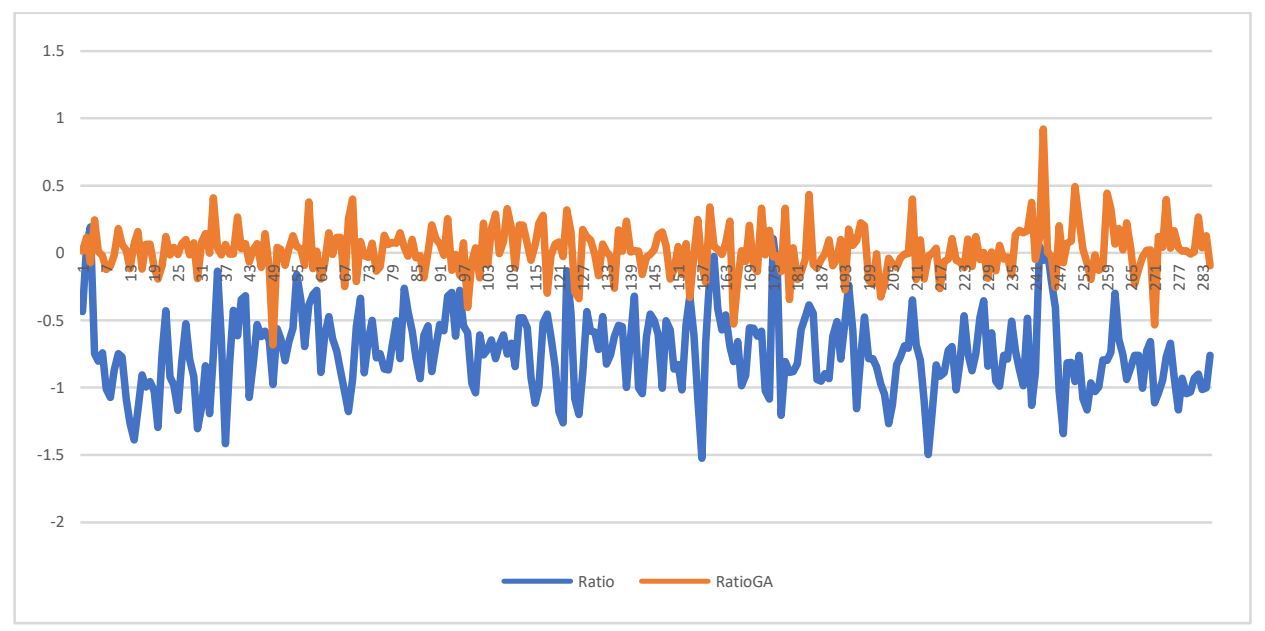

Figure 2. Évolution du Ratio Optimal sur les horizons de couverture d'une fréquence de 2 semaines

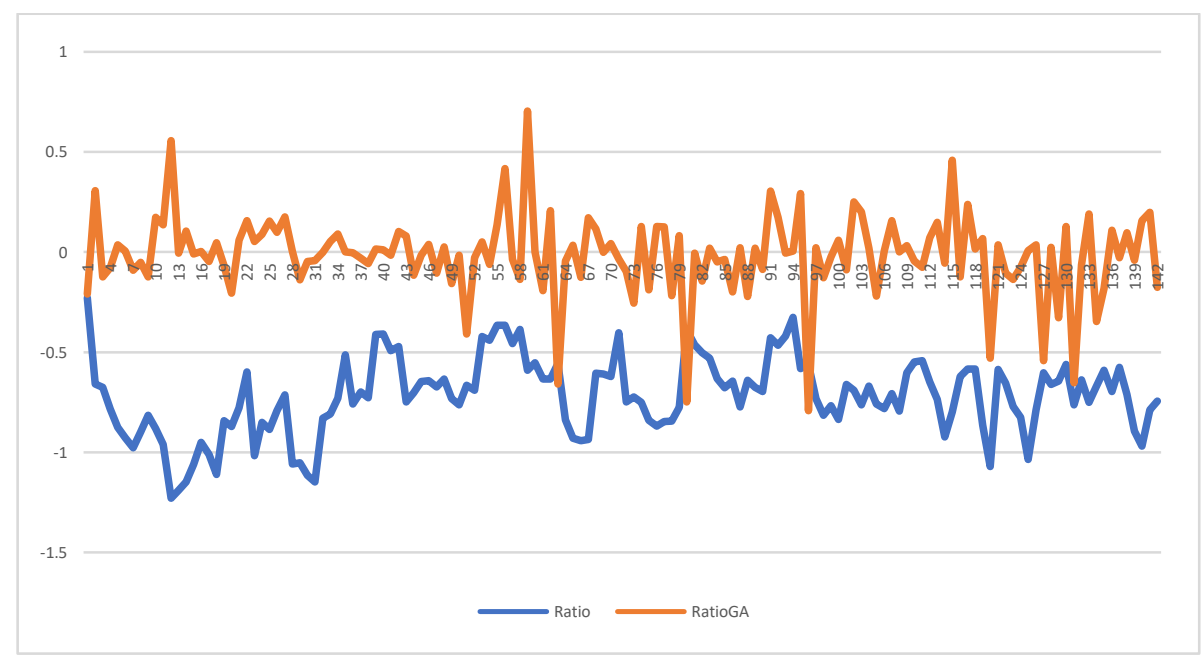

Figure 3. Evolution du Ratio Optimal sur les horizons de couverture d'une fréquence d'un mois

Dans les figures 4 et 5 ci-dessous, nous avons l'évolution de la variance obtenue à partir de la couverture optimale d'Ederington et de l'algorithme GA dans une période de deux semaines et d'un mois. Nous pouvons clairement remarquer qu'en utilisant l'algorithme GA nous étions en mesure de mieux optimiser le risque de change par rapport à celui du taux optimal de la littérature (équation 3). L'évolution de la variance de l'algorithme GA est toujours plus petite que celle de la variance optimale d'Ederington et la différence entre eux devient plus petite à mesure que la fréquence diminue les écarts entre les deux variances est moins remarquable dans fréquence de 2 semaines.

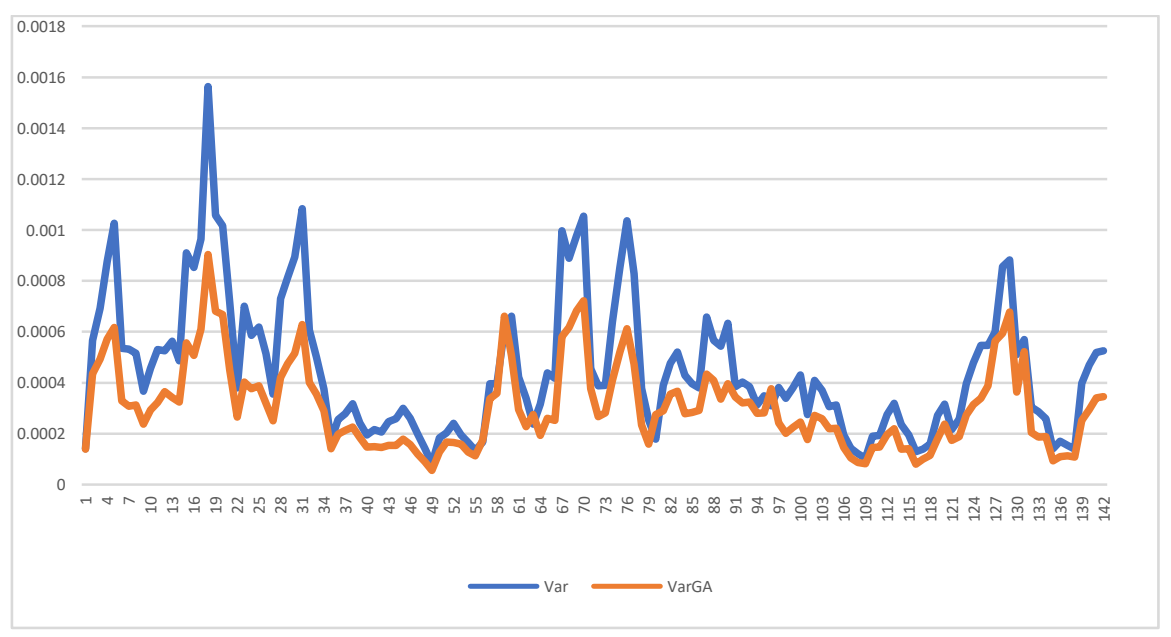

Figure 4. Évolution de la variance sur les horizons de couverture d'un mois 


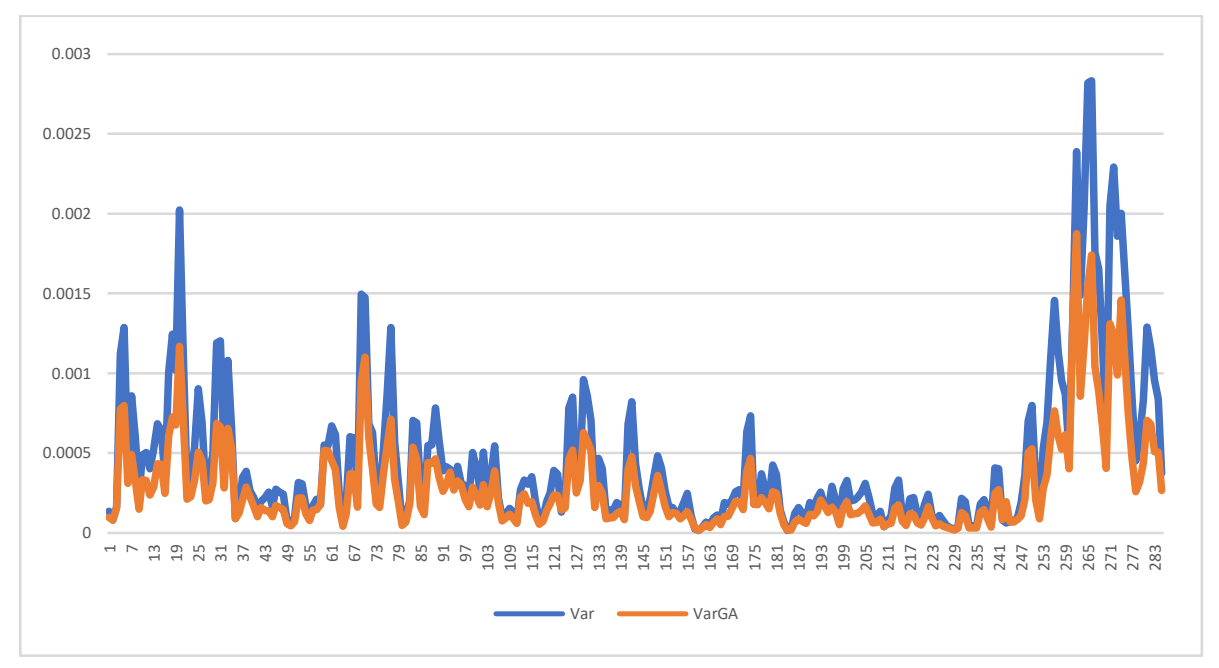

Figure 5. Évolution de la variance sur les horizons de couverture de 2 semaines

Nous pouvons constater que l'algorithme GA nous offre un taux de risque plus petit que celui de la littérature du modèle d'Ederington. Si on veut vraiment investir dans des contrats à terme sur cet exemple d'application du pétrole, on peut obtenir un meilleur ratio que le taux de littérature avec moins de risque. L'algorithme génétique nous a permis d'avoir un risque minimum que celui d'Ederington mais avec un taux qui est imprévisible et dont les valeurs sont plus dispersées. Nous pouvons conclure que la valeur du taux de couverture de GA est aussi variante que le taux optimal de la littérature mais avec un minimum de risque en appliquant l'algorithme GA à la fonction objectif de minimisation de la variance.

\section{Conclusion}

Sur le marché financier international, nous faisant face à plusieurs risques. Le risque de change causé par la fluctuation des prix des matières premières et des devises en fait partie. La volatilité qui est synonyme de risque est normalement réduite par des mesures de couverture comme les contrats à terme que les financiers doivent utiliser de manière optimale pour que la couverture soit efficace voire idéale. L'algorithme génétique, qui est déjà reconnu par sa rapidité et qui se résume sur trois étapes essentielles sélection-crossover-mutation, est proposé dans cette étude comme une technique beaucoup plus rapide et meilleure pour découvrir la méthode optimale pour réduire le risque de change. C'est une approche large de calcul du risque et de détermination du taux le plus approprié pour avoir le risque le plus faible ou le risque que nous ne voulons pas dépasser. Par rapport au taux de couverture optimal d'Ederington, l'algorithme génétique a donné de meilleurs résultats avec une diminution remarquée du risque et plus efficace pour déterminer le ratio optimal de pondération des contrats à terme engagés dans nos propres conditions.

Acknowledgement. Rhouas Sara remercie le Centre National de la Recherche Scientifique et Technologique (CNRST) de lui avoir accordé une bourse nationale de recherche doctorale. 


\section{Bibliographie}

[LIBO 21] LIBO YIN, ZHI SU, MAN LU, « Is oil risk important for commodity-related currency returns? », Research in International Business and Finance, 101604, 2021.

[MIC 22] MiCHAEL KUNKLER, «Hedging local currency risk with precious metals », The North American Journal of Economics and Finance, Vol 59, 101589, 2022.

[AND 21] G. ANDREW KAROLYI, YING WU, « Understanding the pricing of currency risk in global equity markets », Journal of Multinational Financial Management, 100727, 2021.

[DON 05] DONALD LIEN, " The use and abuse of the hedging effectiveness measure », International Review of Financial Analysis, Volume 14, Issue 2, Pages 277-282, 2005.

[BEA 15] BEATRIZ MARTÍNEZ, HIPÒLIT TORRÓ, «European natural gas seasonal effects on futures hedging », Energy Economics, Volume 50, Pages 154-168, 2015.

[SAI 16] Saima Gulzar Ahmad, Chee Sun Liew, Ehsan Ullah Munir, Tan Fong Ang, Samee U. Khan, «A hybrid genetic algorithm for optimization of scheduling workflow applications in heterogeneous computing systems, », Journal of Parallel and Distributed Computing, vol. 87, pp. 80-90, 2016.

[MAH 16] M.J. MAHMOODABADI, A.R. NemATI, « A novel adaptive genetic algorithm for global optimization of mathematical test functions and real-world problems », Engineering Science and Technology, an International Journal, Volume 19, Issue 4, Pages 2002-2021, 2016.

[MAA 16] I. MAATOUK, N. ChebBo, I. JARKASs, E. CHATELET, « Maintenance Optimization using Combined Fuzzy Genetic Algorithm and Local Search », IFAC-PapersOnLine, Volume 49, Issue 12, Pages 757-762, 2016.

[LILI 21] Lili Xu, «Research on computer interactive optimization design of power system based on genetic algorithm», Energy Reports, Volume 7, Supplement 7, Pages 1-13, 2021.

[HYU 19] HYUKGEUN CHOI, JINHYUN KIM, YOURIM YOON, BYUNG-Ro MOON, «Investigation of incremental hybrid genetic algorithm with subgraph isomorphism problem», Swarm and Evolutionary Computation, Volume 49, Pages 75-86, 2019.

[IVAN 19] IVAN VlaŠIĆ, MARKO ĐURASEVIĆ, DOMAGOJ JAKOBOVIĆ,, «Improving genetic algorithm performance by population initialisation with dispatching rules », Computers \& Industrial Engineering, Volume 137, 106030, 2019.

[XUN 11] Xunbo ShuaI, XIangguang Zhou, «A Genetic Algorithm Based on Combination Operators », Procedia Environmental Sciences, Volume 11, Part A, Pages 346-350, 2011.

[TRU 15] Ms. Trupti Bhoskar, Mr. OMkar K. Kulkarni, Mr. Ninad K. KulKarni, Ms. Sujata L. Patekar, G.M. KAKANDIKAR, V.M. NANDEDKAR, « Genetic Algorithm and its Applications to Mechanical Engineering: A Review », Materials Today: Proceedings, Volume 2, Issues 4-5, Pages 2624-2630, 2015.

[FAR 15] FARSHAD KIYOUMARSI, « Mathematics Programming based on Genetic Algorithms Education », Procedia Social and Behavioral Sciences, Volume 192, Pages 70-76, 2015.

[MAR 16] Marco VAnnucci, Valentina Colla, Stefano Dettori, «Fuzzy Adaptive Genetic Algorithm for Improving the Solution of Industrial Optimization Problems », IFAC-PapersOnLine, Volume 49, Issue 12, Pages 1128-1133, 2016.

[MIG 99] MigUEL LACASSE, «Analyse comparative des modèles de couverture pour le risque de change à l'aide de contrats à terme sur devises », Ecole des hautes études commerciales affiliée à l'université de Montréal, Janvier 1999.

[MAN 06] SElim MANKAÏ, CHAKER AlOUI « Performance des modèles d'estimation du ratio de couverture optimal : une nouvelle approche et application au contrat à terme sur CAC $40 »$, Gestion 2000, Volume 23, Numéro 4, Pages 287, 2006.

[STE 18] STEFAN EICHLER, INGMAR ROEVEKAMP, « A market-based measure for currency risk in managed exchange rate regimes », Journal of International Financial Markets, Institutions and Money, Volume 57, Pages 141-159, 2018.

[GOK 21] Goknur BuyukKara, C. Coskun KuCukozMen, E. TOlga Uysal, « Optimal hedge ratios and hedging effectiveness: An analysis of the Turkish futures market », Borsa Istanbul Review, 2021. 
[SUS 16] Susana Álvarez-DíEz, Eva Alfaro-Cid, Matilde O. Fernández-Blanco, « Hedging foreign exchange rate risk: multi-currency diversification », European Journal of Management and Business Economics, Volume 25, Issue 1, Pages 2-7, 2016. 